Proyecciones Journal of Mathematics

Vol. 32, No 4, pp. 321-332, December 2013.

Universidad Católica del Norte

Antofagasta - Chile

\title{
Multiplication and Composition operators on
} $w_{p}(f)$

\author{
Kuldip Raj, Sunil K. Sharma and Seema Jamwal \\ Shri Mata Vaishno Devi University, India \\ Received : June 2012. Accepted : October 2013
}

\begin{abstract}
In this paper we characterize the boundedness, closed range, invertibility of the multiplication operators acting on sequence spaces $w_{p}(f)$ defined by a modulus function. We also make an efforts to study some properties of composition operators on these spaces.
\end{abstract}

Subjclass [2000] : Primary 47B20, Secondary $47 B 38$.

Keywords : Modulus function, multiplication operator, composition operator, closed range, invertibility. 


\section{Introduction and Preliminaries}

A modulus function is a function $f:[0, \infty) \rightarrow[0, \infty)$ such that

1. $f(x)=0$ if and only if $x=0$;

2. $f(x+y) \leq f(x)+f(y)$ for all $x \geq 0, y \geq 0$;

3. $f$ is increasing;

4. $f$ is continuous from right at 0 .

It follows that $f$ must be continuous everywhere on $[0, \infty)$. The modulus function may be bounded or unbounded. For example, if we take $f(x)=\frac{x}{x+1}$, then $f(x)$ is bounded. If $f(x)=x^{p}, 0<p<1$, then the modulus $f(x)$ is unbounded. Subsequently, modulus function has been discussed in ([2], [7], [9]) and many others.

For any sequence $x$, write

$$
d_{m n}=d_{m n}(x)=\frac{1}{m+1} \sum_{i=0}^{m} x_{n+i} .
$$

G. G. Lorentz [6] proved that

$$
\hat{c}=\left\{x: \lim _{m \rightarrow \infty} d_{m n}(x) \text { exists uniformly in } n\right\} .
$$

Khan M. A. [3] extend the definition of $d_{m n}$ to $m=-1$ by taking $d_{-1, n}=x_{n-1}$, then write for $m, n \geq 0$

$$
t_{m n}=t_{m n}(x)=d_{m n}(x)-d_{m-1, n}(x) .
$$

A straight forward calculation then show that

$$
t_{m n}=\frac{1}{m(m+1)} \sum_{v=1}^{m} v\left(x_{n+v}-x_{n+v-1}\right) .
$$

If $f$ is a modulus function and homogeneous of degree 1 , then we define a sequence space as

$$
w_{p}(f)=\left\{x: \sup _{n} \sum_{m} m^{p-1} f\left(\left|t_{m n}(x)\right|^{p}\right)<\infty\right\} .
$$


The space $w_{p}(f)$ with the norm

$$
\|x\|=\sup _{n}\left\{\sum_{m=1}^{\infty} m^{p-1} f\left(\left|t_{m n}(x)\right|^{p}\right)\right\}^{1 / p}, \quad p \geq 1 \text { for } x \in w_{p}(f)
$$

is a Banach space.

Let $v: \mathbf{N} \rightarrow \mathbf{N}$ and $u: \mathbf{N} \rightarrow \mathbf{C}$ be two mappings.

Then the bounded linear transformations

$$
T_{v}: w_{p}(f) \rightarrow w_{p}(f)
$$

and

$$
M_{u}: w_{p}(f) \rightarrow w_{p}(f)
$$

defined by $\left(T_{v} h\right)(x)=h(v(x))$ and $\left(M_{u} h\right)(x)=u(x) h(x)$ are called composition and multiplication operators respectively. By $B\left(w_{p}(f)\right)$, we denote the set of all bounded linear operators from $w_{p}(f)$ into itself and $[z(u)]$ denote, the set $\{n \in \mathbf{N}: u(n)=0\}$. For more details about the study of multiplication and composition operators see ([1], [4], [5], [8], [10], [11]).

In this paper we study multiplication and composition operators acting on sequence spaces $w_{p}(f)$ defined by a modulus function.

\section{Multiplication operators acting on sequence spaces defined by a modulus function}

In this section we characterize multiplication operators acting on $w_{p}(f)$.

Theorem 2.1. Let $M_{u}: w_{p}(f) \rightarrow w_{p}(f)$ be a linear transformation. Then $M_{u}$ is a bounded operator if and only if there exists $M>0$ such that

$$
f\left(\left|u(m) t_{m n}(x)\right|^{p}\right) \leq M f\left(\left|t_{m n}(x)\right|\right)^{p}
$$

for all $m \in N$. 
Proof. Suppose that the condition of the theorem is true. For $x \in$ $w_{p}(f)$, we have

$$
\sup _{n} \sum_{m=1}^{\infty} m^{p-1} f\left(\left|u(m) t_{m n}(x)\right|^{p}\right) \leq M \sup _{n} \sum_{m=1}^{\infty} m^{p-1} f\left(\left|t_{m n}(x)\right|^{p}\right)<\infty
$$

Thus $M_{u} x \in w_{p}(f)$. Further,

$$
\begin{aligned}
\left\|M_{u}(x)\right\| & =\sup _{n}\left\{\sum_{m=1}^{\infty} m^{p-1} f\left(\left|u(m) t_{m n}(x)\right|^{p}\right)\right\}^{1 / p} \\
& \leq M \sup _{n}\left\{\sum_{m=1}^{\infty} m^{p-1} f\left(\left|t_{m n}(x)\right|^{p}\right)\right\}^{1 / p} \\
& \leq M\|x\| .
\end{aligned}
$$

This proves the continuity of $M_{u}$ at the origin and hence everywhere in view of linearity of $M_{u}$.

Conversely, if the condition of the theorem were false, then for every integer $k>0$ there exists $n_{k} \in N$ and $y_{k}=y \in \mathbf{R}^{+}$such that

$$
f\left(\left|u\left(n_{k}\right) t_{m n}\left(y_{k}\right)\right|^{p}\right)>k f\left(\left|t_{m n}\left(y_{k}\right)\right|^{p}\right)
$$

Let $g_{k}=t_{m n}\left(y_{k}\right) \chi_{\left\{n_{k}\right\}}$. Then

$$
\begin{gathered}
k\left\|g_{k}\right\|=k\left\|t_{m n}\left(y_{k}\right) \chi_{\left\{n_{k}\right\}}\right\| \\
=k\left(\sup _{n} \sum_{m=1}^{\infty} m^{p-1} f\left(\left|t_{m n}\left(y_{k}\right)\right|\right)^{p}\right)^{1 / p} \\
<\left(\sup _{n} \sum_{m=1}^{\infty} m^{p-1} f\left(\left|u\left(n_{k}\right) t_{m n}\left(y_{k}\right)\right|\right)^{p}\right)^{1 / p} \\
=\left(\sup _{n} \sum_{m=1}^{\infty} m^{p-1} f\left|M_{u} g_{k}\right|^{p}\right)^{1 / p} \\
=\left\|M_{u} g_{k}\right\| .
\end{gathered}
$$

This proves that $M_{u}$ is not bounded. Hence the condition must be true. 
Theorem 2.2. Let $A M_{u}=M_{u} A$. Then $A$ is a multiplication operator.

Proof. Let $V=A e$. Then

$$
A e_{n}=A M_{e_{n}} e=M_{e_{n}} A e=M_{e_{n}} V=e_{n} V=V e_{n}=M_{V} e_{n} .
$$

We now prove that $V$ induces a multiplication operator. If $V$ does not induce a bounded operator, then for every $k \in N$, there exists $n_{k} \in N$ such that

$$
f\left(\left|V\left(n_{k}\right) t_{m n}\left(y_{k}\right)\right|^{p}\right)>m f\left(\left|t_{m n}\left(y_{k}\right)\right|^{p}\right) .
$$

Let $g_{k}=t_{m n}\left(y_{k}\right) e_{n_{k}}$. Then

$$
\begin{aligned}
k\left\|g_{k}\right\| & =k\left\|t_{m n}\left(y_{k}\right) e_{n_{k}}\right\| \\
& =k\left(\sup _{n} \sum_{m=1}^{\infty} m^{p-1} f\left(\left|t_{m n}\left(y_{k}\right)\right|\right)^{p}\right)^{1 / p} \\
& <\left(\sup _{n} \sum_{m=1}^{\infty} m^{p-1} f\left(\left|V\left(n_{k}\right) t_{m n}\left(y_{k}\right)\right|\right)^{p}\right)^{1 / p} \\
& =\left(\sup _{n} \sum_{m=1}^{\infty} m^{p-1} f\left|A g_{k}\right|^{p}\right)^{1 / p} \\
& =\left\|A g_{k}\right\|,
\end{aligned}
$$

which contradicts the continuity of $A$. Hence $A$ must be a bounded operator and $A=M_{V}$.

Theorem 2.3. Let $M_{u} \in B\left(w_{p}(f)\right)$. Then $M_{u}$ is invertible if and only if there exists $\epsilon>0$ such that

$$
f\left(\left|u(k) t_{m n}(y)\right|^{p}\right) \geq \epsilon f\left(\left|t_{m n}(y)\right|^{p}\right), \quad \forall p \in \mathbf{N} \text { and } y \in \mathbf{R}^{+} .
$$

Proof. We first assume that there exists $\epsilon>0$ such that

$$
f\left(\left|u(k) t_{m n}(y)\right|^{p}\right) \geq \epsilon f\left(\left|t_{m n}(y)\right|^{p}\right), \quad \forall p \in \mathbf{N} \text { and } y \in \mathbf{R}^{+} .
$$

Now 


$$
\begin{gathered}
\epsilon f\left[\frac{\left|t_{m n}(y)\right|^{p}}{|u(k)|^{p}}\right] \leq f\left[|u(k)|^{p} \cdot \frac{\left|t_{m n}(y)\right|^{p}}{|u(k)|^{p}}\right] \\
=\mathrm{f}\left(-\left.\mathrm{t}_{m n}(y)\right|^{p}\right) \text { or } \\
f\left[\left|\frac{1}{|u(k)|^{p}} t_{m n}(y)\right|^{p}\right] \leq \frac{1}{\epsilon} f\left(\left|t_{m n}(y)\right|^{p}\right), \quad \forall p \in \mathbf{N} .
\end{gathered}
$$

This proves that $M_{V}$ is a bounded operator, where $V=\frac{1}{u}$. Clearly $M_{V}$ is inverse of $M_{u}$.

Conversely, suppose that $M_{u}$ is invertible with $M_{V}$ as its inverse. Clearly $V=\frac{1}{u}$. Hence by continuity of $M_{V}$, there exists $M>0$ such that

$$
f\left(\left|V(k) t_{m n}(y)\right|^{p}\right) \leq M f\left(\left|t_{m n}(y)\right|^{p}\right), \quad \forall k \in \mathbf{N} \text { and } y \in \mathbf{R}^{+} .
$$

Or equivalently

$$
f\left[\left|\frac{1}{|u(k)|} t_{m n}(y)\right|^{p}\right] \leq M f\left(\left|t_{m n}(y)\right|^{p}\right)
$$

Taking

$$
\left|t_{m n}(y)\right|=\left|u(k) t_{m n}(y)\right|
$$

we get

$$
f\left(\left|t_{m n}(y)\right|^{p}\right) \leq M f\left(\left|u(k) t_{m n}(y)\right|^{p}\right)
$$

or

$$
f\left(\left|u(k) t_{m n}(y)\right|^{p}\right) \geq \frac{1}{M} f\left(\left|t_{m n}(y)\right|^{p}\right) \forall k \in \mathbf{N} .
$$

Taking $\epsilon=\frac{1}{M}$, we get

$$
f\left(\left|u(k) t_{m n}(y)\right|^{p}\right) \geq \epsilon f\left(\left|t_{m n}(y)\right|^{p}\right) .
$$

Hence the condition must be true.

Theorem 2.4. Let $M_{u} \in B\left(w_{p}(f)\right)$. Then $M_{u}$ is Fredholm if and only if 
(i) $[Z(u)]$ is a finite set

(ii) there exists $\epsilon>0$ such that

$$
f\left(\left|u(k) t_{m n}(y)\right|^{p}\right) \geq \epsilon f\left(\left|t_{m n}(x)\right|^{p}\right) \forall m \in[Z(u)]^{\prime} .
$$

Proof. If $[Z(u)]$ is a finite set, then $\operatorname{ker} M_{u}$ is finite dimensional. From the condition (ii), $M_{u}$ has closed range.

Moreover $\operatorname{dim}\left(w_{p}(f) / \operatorname{ran} M_{u}\right)$ is finite. This proves that $M_{u}$ is Fredholm.

The converse of the theorem is obvious.

Corollary 2.5. Let $M_{u} \in B\left(w_{p}(f)\right)$. Then $M_{u}$ has closed range if and only if there exists $\delta>0$ such that

$$
f\left(\left|u(k) t_{m n}(y)\right|^{p}\right) \geq \delta f\left(\left|t_{m n}(y)\right|^{p}\right), \quad \forall k \in[Z(u)]^{\prime} \text { and } y \in \mathbf{R}^{+} .
$$

Proof. Assume that the condition of the theorem is true. Let $h \in \overline{\operatorname{ran} M_{u}}$.

Then there exists a sequence $\left\{h_{n}\right\}$ such that $M_{u} h_{n} \rightarrow h$ that is $\| M_{u} h_{n}-$ $M_{u} h \| \rightarrow 0$ as $n \rightarrow \infty$. Now $\left\{M_{u} h_{n}\right\}$ is a Cauchy sequence. Therefore for every $\epsilon>0$ there exists $n_{0} \in N$ such that

$$
\left\|M_{u} t_{m n} h_{n}-M_{u} t_{m n} h_{k}\right\|<\epsilon \forall n, k \geq n_{0} .
$$

Now

$$
\begin{aligned}
\delta \sup _{n \in[Z(u)]^{\prime}} \sum_{m=1}^{\infty} m^{p-1} f\left(\left|t_{m n}\left(h_{n}-h_{k}\right)\right|^{p}\right) & \leq \sup _{n \in[Z(u)]^{\prime}} \sum_{m=1}^{\infty} m^{p-1} f\left(\left|u(m) t_{m n}\left(h_{n}-h_{k}\right)\right|^{p}\right) \\
& <\epsilon \quad \forall n, k \geq n_{0} .(1)
\end{aligned}
$$

Define

$$
\tilde{h_{n}}(k)= \begin{cases}h_{n}(k), & \text { if } m \in[Z(u)]^{\prime} \\ 0, & \text { elsewhere. }\end{cases}
$$

Then from (1) it follows that $\left\{\tilde{h_{n}}\right\}$ is a Cauchy sequence in $w_{p}(f)$. But $w_{p}(f)$ is complete. 
Therefore there exists $\tilde{h} \in w_{p}(f)$ such that $\tilde{h_{n}} \rightarrow \tilde{h}$. Hence by continuity of $M_{u}$, we get $M_{u} h_{n}=M_{u} \tilde{h_{n}} \rightarrow M_{u} \tilde{h}$. Hence $h=M_{u} \tilde{h}$ so that $h \in \operatorname{ran} M_{u}$. Thus $M_{u}$ has closed range.

Conversely, if the condition of the theorem were false, then for every positive integer $k$ there exists $n_{k} \in N$ and $y_{k} \in \mathbf{R}^{+}$such that

$$
f\left(\left|u\left(n_{k}\right) t_{m n}\left(y_{k}\right)\right|^{p}\right)<1 / k f\left(\left|t_{m n} y_{k}\right|^{p}\right) .
$$

Let $g_{k}=t_{m n} y_{k} \chi_{\left\{n_{k}\right\}}$.

Then

$$
\begin{aligned}
& \qquad|| M_{u} g_{k}||=\sup _{n}\left\{\sum_{m=1}^{\infty} m^{p-1} f\left(\left|u \cdot g_{k}\right|^{p}\right)\right\}^{1 / p} \\
& =\sup _{n}\left\{\sum_{m=1}^{\infty} m^{p-1} f\left(\left|u\left(n_{k}\right) t_{m n}\left(y_{k}\right)\right|^{p}\right)\right\}^{1 / p} \\
& \leq 1 / k \sup _{n}\left\{\sum_{m=1}^{\infty} m^{p-1} f\left(\left|t_{m n}\left(y_{k}\right)\right|^{p}\right)\right\}^{1 / p} \\
& =1 / \mathrm{k}\left\|g_{k}\right\| .
\end{aligned}
$$

This proves that $M_{u}$ is not bounded away from zero so that $M_{u}$ does not have closed range.

\section{Composition operators acting on sequence spaces defined by a modulus function}

In this section we study some properties of composition operators on $w_{p}(f)$.

Theorem 3.1. Let $T_{v}: w_{p}(f) \rightarrow w_{p}(f)$ be a linear transformation. Then $T_{v}$ is a bounded operator if there exists $M>0$ such that

$$
\sum_{k \in v^{-1}(n)} m^{p-1} f\left(\left|t_{m k}(x)\right|^{p}\right) \leq M m^{p-1} f\left(\left|t_{m n}(x)\right|^{p}\right) .
$$


Proof. Suppose that the condition of the theorem is true. If $x \in w_{p}(f)$, then

$$
\sup _{n} \sum_{m=1}^{\infty} \sum_{k \in v^{-1}(n)} m^{p-1} f\left(\left|t_{m k}(x)\right|^{p}\right) \leq M \sup _{n} \sum_{m=1}^{\infty} m^{p-1} f\left(\left|t_{m n}(x)\right|^{p}\right)
$$

$<\infty$, which shows that $T_{v} x \in w_{p}(f)$. Further,

$$
\begin{gathered}
\qquad \mid\left\|T_{v} x\right\|_{f}=\sup _{n}\left\{\sum_{m=1}^{\infty} m^{p-1} f\left(\left|t_{m n}(x \circ v(k))\right|^{p}\right)\right\}^{1 / p} \\
=\sup _{n}\left\{\sum_{m=1}^{\infty} \sum_{k \in v^{-1}(n)} m^{p-1} f\left(\left|t_{m k} x\right|^{p}\right)\right\}^{1 / p} \\
\leq M \sup _{n}\left\{\sum_{m=1}^{\infty} m^{p-1} f\left(\left|t_{m n}(x)\right|^{p}\right)\right\}^{1 / p} \\
\leq M\|x\|_{f} \cdot(2)
\end{gathered}
$$

The continuity of $T_{v}$ at origin follows from the inequality (2). Since $T_{v}$ is linear, so it is continuous everywhere.

Theorem 3.2. Let $T_{v} \in B\left(w_{p}(f)\right)$. Then $T_{v}$ has closed range if there exists $\delta>0$ such that

$$
\sum_{k \in v^{-1}(n)} m^{p-1} f\left(\left|t_{m k}(x)\right|^{p}\right) \geq \delta m^{p-1} f\left(\left|t_{m n}(x)\right|^{p}\right) \text { for every } m \in \mathbf{N} .(3)
$$

Proof. We assume that the condition (3) is true. We have to show that $T_{v}$ has closed range. Let $x \in \overline{\operatorname{ran} T_{v}}$ and let $\left\{x^{i}\right\}$ be a sequence in $w_{p}(f)$ such that $T_{v} x^{n} \rightarrow x$. Then for every $\epsilon>0$ there exists positive integer $n_{0}$ such that

$$
\left\|T_{v} x^{i}-T_{v} x^{j}\right\|<\epsilon \forall i, j \geq n_{0} .
$$

Equivalently,

$$
\epsilon>\sup _{n}\left\{\sum_{m=1}^{\infty} \sum_{k \in v^{-1}(n)} m^{p-1} f\left(\left|t_{m k}\left(x^{i} \circ v(k)-x^{j} \circ v(k)\right)\right|^{p}\right)\right\}^{1 / p}
$$




$$
\begin{aligned}
& \geq \delta \sup _{n}\left\{\sum_{m=1}^{\infty} m^{p-1} f\left(\left|t_{m n}\left(x^{i}-x^{j}\right)\right|^{p}\right)\right\}^{1 / p} \\
& =\delta\left\|x^{i}-x^{j}\right\|, \quad \forall i, j \geq n_{0}(4)
\end{aligned}
$$

from (4) it follows that $\left\{x^{i}\right\}$ is a Cauchy sequence in $w_{p}(f)$. In view of completeness of $w_{p}(f)$, there exists $y \in w_{p}(f)$ such that $x^{i} \rightarrow y$. From the continuity of $T_{v}, T_{v} x^{i} \rightarrow T_{v} y$. Hence $x=T_{v} y$ so that $x \in \operatorname{ran} T_{v}$. Hence ran $T_{v}$ is closed.

Theorem 3.3. Let $T_{v} \in B\left(w_{p}(f)\right)$. Then $T_{v}$ is an isometry if

$$
\sum_{k \in v^{-1}(n)} m^{p-1} f\left(\left|t_{m k}(x)\right|^{p}\right)=m^{p-1} f\left(\left|t_{m n}(x)\right|^{p}\right) .
$$

Proof. If the condition of the theorem is satisfied, then for every $x \in$ $w_{p}(f)$, we have

$$
\begin{aligned}
\left\|T_{v} x\right\| & =\sup _{n}\left\{\sum_{m=1}^{\infty} \sum_{k \in v^{-1}(m)} m^{p-1} f\left(\left|t_{m k} x\right|^{p}\right)\right\}^{1 / p} \\
& =\sup _{n}\left\{\sum_{m=1}^{\infty} m^{p-1} f\left(\left|t_{m n}(x)\right|^{p}\right)\right\}^{1 / p} \\
& =\|x\| .
\end{aligned}
$$

Hence $T_{v}$ is an isometry.

Theorem 3.4. Let $T_{v} \in B\left(w_{p}(f)\right)$. If $T_{v}$ is an isometry, then

$$
\sup _{n} \sum_{k \in v^{-1}(m)} k^{p-1} f\left(\left|t_{n k}(x)\right|^{p}\right)=\sup _{n} m^{p-1} f\left(\left|t_{m n} x\right|^{p}\right) .
$$

Proof. The proof is trivial. 


\title{
References
}

[1] M. B. Abrahmse, Multiplication operators, Hilbert space operators. Lecture notes in Mathematics, 693 : pp. 17-36, (1978).

[2] T. Bilgen, On statistical convergence. An. Univ. Timisoara Ser. Math. Inform., 32 : pp. 3-7, (1994).

[3] M. A. Khan, Some sequence spaces with an index defined by a modulus function. Thai J. Math., 2 : 259-264, (2004).

[4] B. S. Komal and Kuldip Raj, Multiplication operators induced by operator valued maps. Int. J. Contemp. Math. Sci., Vol.3 : pp. 667$673,(2008)$.

[5] B. S. Komal and P. S. Singh, Composition operators on the space of entire functions. Kodai Math. J., 14 : pp. 463-469, (1991).

[6] G. G. Lorentz, A contribution to the theory of divergent series. Acta. Math., 80 : pp. 167-190, (1948).

[7] E. Malkowsky and E. Savas, Some $\lambda$-sequence spaces defined by a modulus. Arch. Math., 36 : pp. 219-228, (2000).

[8] Kuldip Raj, B. S. Komal and Vinay Khosla, Composition operators on sequence spaces of entire functions. Int. Electron. J. Pure Appl. Math., 1 : pp. 469-474, (2010).

[9] E. Savas, On some generalized sequence spaces defined by a modulus. Indian J. pure Appl. Math., 30 : pp. 459-464, (1999).

[10] R. K. Singh and J. S. Manhas, Composition operators on function spaces. North-Holland, (1993).

[11] H. Takagi and K. Yokouchi, Multiplication and composition operators between $L^{p}$-spaces. Contemp. Math., 232 : pp. 321-338, (1999).

\author{
Kuldip Raj \\ School of Mathematics \\ Shri Mata Vaishno Devi University \\ Katra-182320, J\&K, \\ India \\ e-mail : kuldipraj68@gmail.com
}




\section{Sunil K. Sharma}

School of Mathematics

Shri Mata Vaishno Devi University

Katra-182320, J\&K,

India

e-mail : sunilksharma42@yahoo.co.in

and

\section{Seema Jamwal}

School of Mathematics

Shri Mata Vaishno Devi University

Katra-182320, J\&K,

India

e-mail : seemajamwal8@gmail.com 